\title{
XPO1 expression worsens the prognosis of unfavorable DLBCL that can be effectively targeted by selinexor in the absence of mutant p53
}

Manman Deng ${ }^{1,2+}{ }^{2}$, Mingzhi Zhang ${ }^{3 \dagger}$, Zijun Y. Xu-Monette ${ }^{2 \dagger} \oplus$, Lan V. Pham ${ }^{4 \dagger}$, Alexandar Tzankov ${ }^{5}$, Carlo Visco ${ }^{6}$, Xiaosheng Fang ${ }^{2}$, Govind Bhagat ${ }^{7}$, Feng Zhu², Karen Dybkaer ${ }^{8}$, April Chiu' ${ }^{9}$, Wayne Tam ${ }^{10}$, Youli Zu ${ }^{11}$, Eric D. Hsi ${ }^{12}$, William W. L. Choi ${ }^{13}$, Jooryung Huh ${ }^{14}$, Maurilio Ponzoni ${ }^{15}$, Andrés J. M. Ferreri ${ }^{16}$, Michael B. Møller ${ }^{16}$, Benjamin M. Parsons ${ }^{17}$, J. Han van Krieken ${ }^{18}$, Miguel A. Piris ${ }^{19}$, Jane N. Winter ${ }^{20}$, Fredrick Hagemeister ${ }^{21}$,

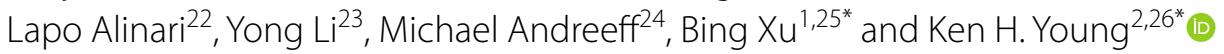

\begin{abstract}
The XPO1 inhibitor selinexor was recently approved in relapsed/refractory DLBCL patients but only demonstrated modest anti-DLBCL efficacy, prompting us to investigate the prognostic effect of XPO1 in DLBCL patients and the rational combination therapies in high-risk DLBCL. High XPO1 expression (XPO1 ${ }^{\text {high }}$ ) showed significant adverse prognostic impact in 544 studied DLBCL patients, especially in those with BCL2 overexpression. Therapeutic study in $30 \mathrm{DLBCL}$ cell lines with various molecular and genetic background found robust cytotoxicity of selinexor, especially in cells with $B C L 2$-rearranged $\left(B C L 2-\mathrm{R}^{+}\right)$DLBCL or high-grade B-cell lymphoma with MYC/BCL2 double-hit (HGBCL-DH). However, expression of mutant (Mut) p53 significantly reduced the cytotoxicity of selinexor in overall cell lines and the BCL2-R and HGBCL-DH subsets, consistent with the favorable impact of XPO ${ }^{\text {high }}$ observed in Mut-p53-expressing patients. The therapeutic effect of selinexor in HGBCL-DH cells was significantly enhanced when combined with a BET inhibitor INCB057643, overcoming the drug resistance in Mut-p53-expressing cells. Collectively, these data suggest that XPO1 worsens the survival of DLBCL patients with unfavorable prognostic factors such as BCL2 overexpression and double-hit, in line with the higher efficacy of selinexor demonstrated in BCL2-R ${ }^{+}$DLBCL and HGBCL-DH cell lines. Expression of Mut-p53 confers resistance to selinexor treatment, which can be overcome by combined INCB057643 treatment in HGBCL-DH cells. This study provides insight into the XPO1 significance and selinexor efficacy in DLBCL, important for developing combination therapy for relapsed/refractory DLBCL and HGBCL-DH.
\end{abstract}

Keywords: XPO1, DLBCL, HGBCL, TP53 mutation, Selinexor, MYC, BCL2

\footnotetext{
*Correspondence: xubingzhangjian@126.com; ken.young@duke.edu ${ }^{\dagger}$ Manman Deng, Mingzhi Zhang, Zijun Y. Xu-Monette and Lan V. Pham have contributed equally to this manuscript.

1 Department of Hematology, The First Affiliated Hospital of Xiamen

University and Institute of Hematology, Xiamen University, School of Medicine, Xiamen, Fujian, China

2 Division of Hematopathology, Department of Pathology, Duke University Medical Center, Durham, NC 27710, USA

Full list of author information is available at the end of the article
}

\section{To the editor}

$\mathrm{XPO1}$ (exportin 1) is a well-characterized nuclear export protein responsible for the nuclear-cytoplasmic transport and cellular homeostasis of up to 220 cargoes, including the tumor suppressors p53 and ІкB $[1,2]$. Abnormal XPO1 expression correlates with worse prognoses in human malignancies. Targeting XPO1 is a promising therapeutic approach in cancer $[1,2]$. The XPO1 original author(s) and the source, provide a link to the Creative Commons licence, and indicate if changes were made. The images or other third party material in this article are included in the article's Creative Commons licence, unless indicated otherwise in a credit line to the material. If material is not included in the article's Creative Commons licence and your intended use is not permitted by statutory regulation or exceeds the permitted use, you will need to obtain permission directly from the copyright holder. To view a copy of this licence, visit http://creativecommons.org/licenses/by/4.0/. The Creative Commons Public Domain Dedication waiver (http://creativeco mmons.org/publicdomain/zero/1.0/) applies to the data made available in this article, unless otherwise stated in a credit line to the data. 
inhibitor selinexor has received FDA approval recently to treat refractory/relapsed $(\mathrm{R} / \mathrm{R})$ diffuse large $\mathrm{B}$-cell lymphoma (DLBCL) after at least 2 lines of systemic therapy, showing an overall response rate of $28 \%$ in the SADAL trial [3]. However, it remains largely unknown whether and how XPO1 interplays with other adverse predictors in DLBCL, how to predict selinexor effectiveness, and what combination therapy is optimal in R/R DLBCL patients. Here, we evaluated the prognostic significance of XPO1 expression in 544 well-characterized DLBCL cases, and investigated the therapeutic effect of selinexor in 30 DLBCL cell lines with variable genetic background.

Patients and Methods for this study are detailed in Additional file 1. XPO1 expression was observed in 217 of 544 (40\%) DLBCL patients with a mean level of $24 \%$.
High level of XPO1 expression $\left(\mathrm{XPO}^{\text {high }}{ }^{\text {; }}>30 \%\right)$ predicted significantly poor progressive-free survival (PFS) and overall survival (OS) in DLBCL patients (Fig. 1a). DLBCL is classified into prognostic favorable germinal center B-cell-like (GCB) and unfavorable activated B-celllike $(A B C)$ subtypes [4]. XPO1 $1^{\text {high }}$ significantly shortened the PFS/OS in ABC-DLBCL but not GCB-DLBCL (Additional file 1: Figure $\mathrm{S} 1 \mathrm{~A}-\mathrm{B}) . \mathrm{XPO} 1^{\text {high }}$ showed significant association with $\mathrm{p} 53$ overexpression $\left(\mathrm{p} 53^{+}\right)$and dual $\mathrm{p} 53^{+} \mathrm{MYC}^{\text {high }}$ expression but not clinical features (Additional file 1: Table S1), unlike a previous study using a different scoring system for XPO1 expression in 131 DLBCL patients [5].

Whether $\mathrm{XPO}^{\text {high }}$ interacts with other adverse prognostic factors and whether XPO1 is a potential
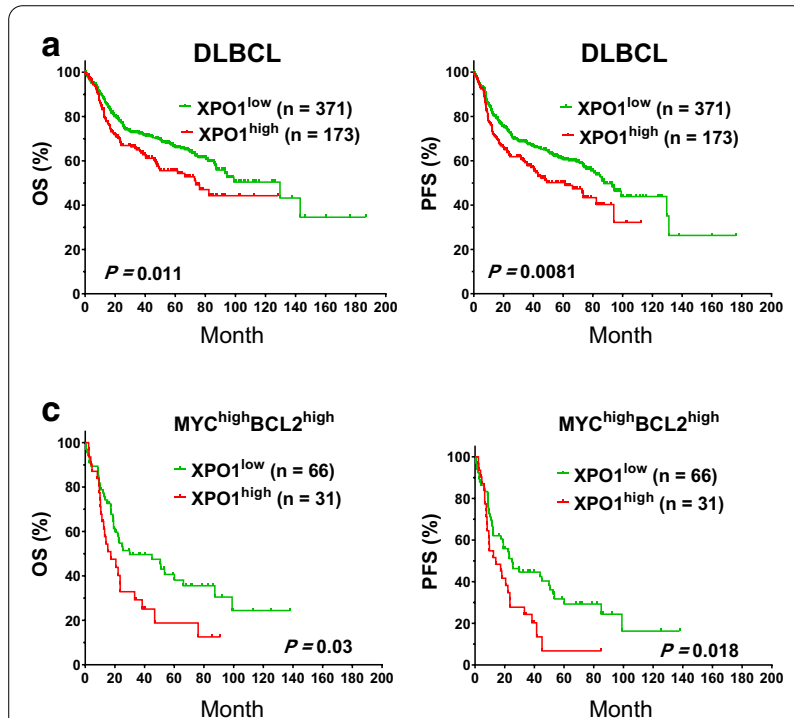

e
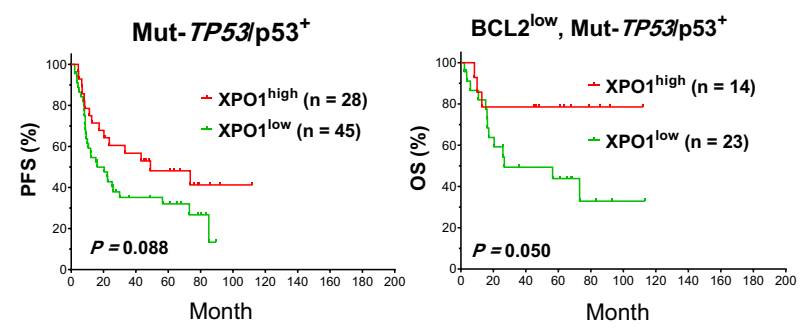
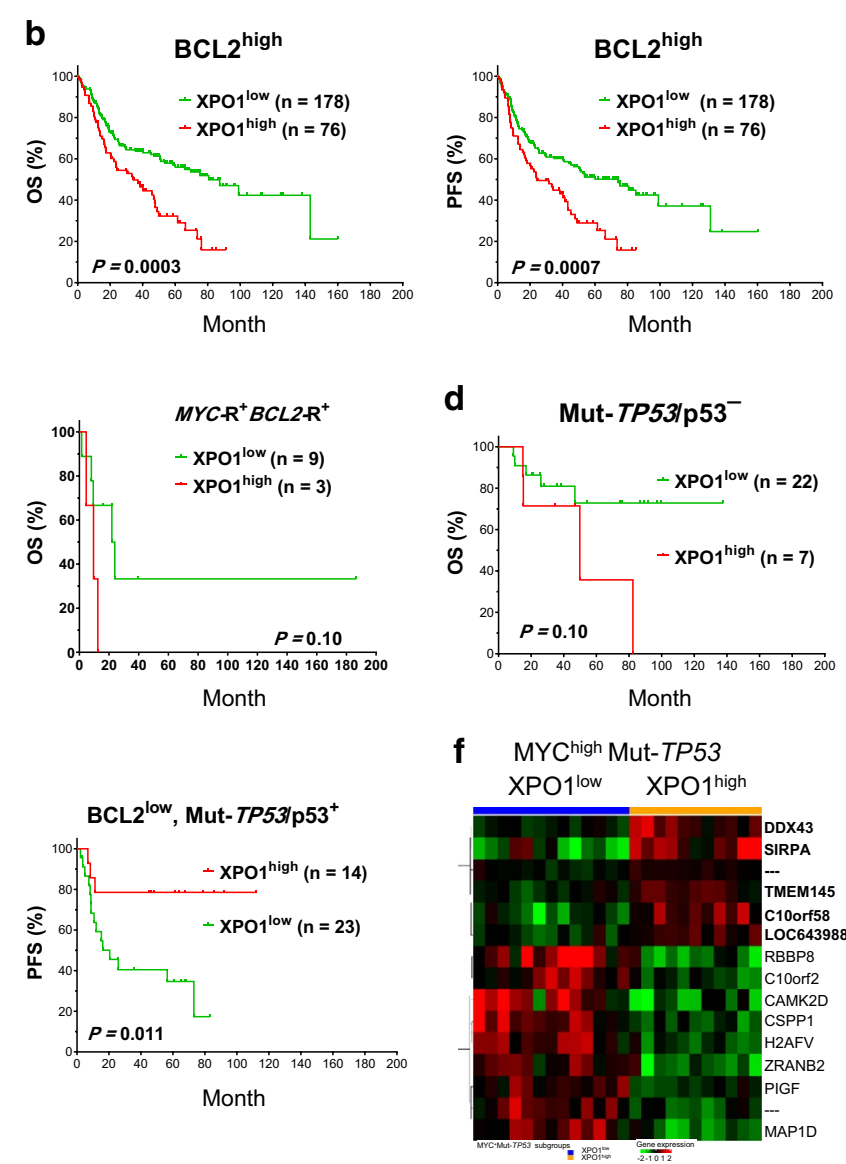

Fig. 1 Impact of XPO1 expression on patient survival in DLBCL. a In the entire cohort, DLBCL patients with high level of XPO1 expression (XPO1 high) had significantly worse OS and PFS than those with low or negative XPO1 expression (XPO1 ${ }^{\text {low }}$ ). b XPO1 ${ }^{\text {high }}$ remarkably worsened the OS/PFS of DLBCL patients with BCL2 high expression. $\mathbf{c} X P O 1^{\text {high }}$ significantly worsened the OS/PFS of patients with dual MYC ${ }^{\text {high }} \mathrm{BCL} 2^{\text {high }}$ expression, and showed a trend of unfavorable effect on OS in patients with dual MYC/BCL2 rearrangements (MYC- $\left.R^{+} B C L 2-R^{+}, H G B C L-D H\right)$. d In TP53-mutated (Mut) DLBCL patients without Mut-p53 overexpression, XPO1 $1^{\text {high }}$ showed a trend of unfavorable prognostic effect on OS. e In Mut-TP53 DLBCL patients with Mut-p53 overexpression, XPO1 ${ }^{\text {high }}$ showed favorable prognostic effect, which was not significant in overall patients but significant in the subset with low BCL2 expression. $\mathbf{f}$ Significantly differentially expressed genes between XPO $1^{\text {high }}$ and XPO1 ${ }^{\text {low }}$ patients with concurrent Mut-TP53 and MYChigh 
therapeutic target in high-risk DLBCL patients were further examined. $\mathrm{XPO}^{\text {high }}$ remarkably worsened the OS and PFS of DLBCL with BCL2 ${ }^{\text {high }}$ or dual $\mathrm{MYC}^{\text {high }} \mathrm{BCL} 2^{\text {high }}$ expression (Fig. 1b,c), which is known as double-expressor lymphoma with unfavorable prognosis [6]. Trends of adverse impact were also observed on PFS in $M Y C$-rearranged $\left(\mathrm{R}^{+}\right)$patients $(P=0.097$; Additional file 1: Figure $\mathrm{S} 1 \mathrm{C})$ and $\mathrm{OS}$ in patients with dual $M Y C-\mathrm{R}^{+} B C L 2-\mathrm{R}^{+}$(Fig. 1c) with dismal prognosis, defined as high-grade B-cell lymphoma with $M Y C / B C L 2$ double-hit (HGBCL-DH) [7]. In patients with TP53 mutation (Mut-TP53) [8], XPO1 ${ }^{\text {high }}$ showed opposite prognostic effects in patients with and without Mut-p53 protein overexpression [9], suggesting the nuclear export may attenuate the oncogenic gain-of-function of Mutp53. In contrast to the negative impact of $X P O 1^{\text {high }}$ in
Mut-TP53/p53-negative patients (Fig. 1d) and in TP53wild type (Wt-TP53) patients (Additional file 1: Figure S1D), a favorable effect was associated with $\mathrm{XPO}^{\text {high }}$ in Mut-TP53/p53-positive patients, which was significant in the BCL2 ${ }^{\text {low }}$ subset (Fig. 1e). Gene expression profiling [4] analysis identified a distinct gene expression signature for $\mathrm{XPO} 1^{\text {high }}$ in patients with Mut-TP53 and MYC $\mathrm{C}^{\text {high }}$ (Fig. 1f), including upregulation of SIRPA, which encodes SIRP $\alpha$, a receptor for CD47 transmitting "do not eat me" signal in phagocytosis, and downregulation of several genes related to DNA repair, metabolism, splicing, or biosynthesis (Additional file 1: Table S2).

Next, selinexor was assessed in 30 DLBCL cell lines, which resulted in significantly reduced cell viability with varying IC50 values (Fig. 2a). ABC-DLBCL and GCB-DLBCL cells were similarly vulnerable to selinexor

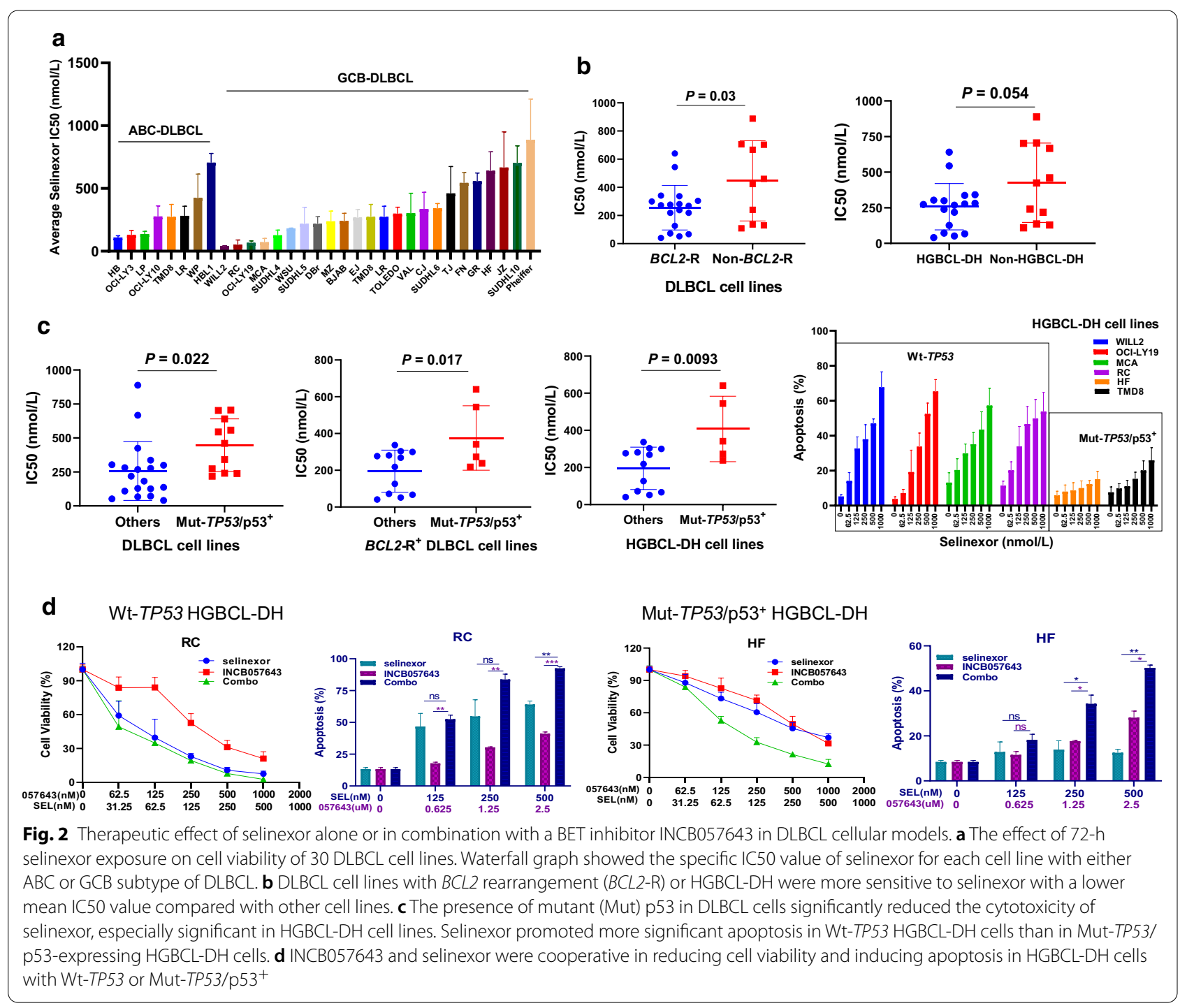


(Additional file 1: Figure S1E), consistent with results in the SADAL clinical trial [3]. Biomarkers significantly associated with higher sensitivity (lower IC50) to selinexor cytotoxicity included $B C L 2-\mathrm{R}$ and HGBCL-DH (Fig. 2b) but not $M Y C-\mathrm{R}$. In contrast, presence of MutTP53/p53 ${ }^{+}$significantly reduced the anti-lymphoma efficacy of selinexor, especially in HGBCL-DH cells (Fig. 2c; Additional file 1: Figure S1F).

Limited efficacy of selinexor in HGBCL with MutTP53/p53 ${ }^{+}$calls for combination strategy. Previous studies showed the synergy between selinexor and venetoclax in DLBCL and double-hit lymphoma $[10,11]$. However, in the SADAL trial [3], patients with MYC ${ }^{\text {high }}$ (but not $B C L 2^{\text {high }}$ ) expression had a lower overall response rate than those without. MYC expression can be inhibited by targeting the bromodomain and extra-terminal domain (BET) proteins [12]. We therefore combined selinexor with a novel BET inhibitor INCB057643. Synergistic effect was observed in DLBCL/HGBCL cells, especially in HGBCL-DH cells with Mut-TP53/p53 ${ }^{+}$(Fig. 2d), supporting INCB057643/selinexor combination as a therapeutic option for HGBCL-DH patients.

In summary, this study demonstrates that $\mathrm{XPO}^{\text {high }}$ is a valuable biomarker in DLBCL with unfavorable prognostic factors, predictive of significantly poorer outcomes in ABC-DLBCL, BCL2 $2^{\text {high }}$ DLBCL, and double-expressor lymphoma but not Mut-p53-expressing DLBCL. Targeting XPO1 with selinexor is similarly effective in GCB-DLBCL and ABC-DLBCL cells, and remarkably effective in $B C L 2-\mathrm{R}^{+}$DLBCL and HGBCL cells without Mut-TP53/p53-positivity. In DLBCL/HGBCL cells, Mut-TP53/p53-positive expression predicts resistance to selinexor. INCB057643 synergizes with selinexor in HGBCL-DH cells, overcoming resistance in Mut-TP53/ p53-positive HGBCL-DH. These findings warrant future investigation on the role of XPO1, selinexor, and combined BET inhibition in R/R DLBCL and HGBCL-DH.

\section{Supplementary information}

Supplementary information accompanies this paper at https://doi. org/10.1186/s13045-020-00982-3.

Additional file 1. Table S1: Clinicopathologic and molecular characteristics of DLBCL patients with high or low XPO1 expression. Table S2: Significantly differentially expressed genes between $\mathrm{XPO} 1^{\text {high }}$ and XPO $1^{\text {low }}$ DLBCL patients with concurrent TP53 mutation and high MYC expression. Figure S1: Biomarker study for XPO1 and selinexor. (A-B) XPO1 ${ }^{\text {high }}$ expression showed significant adverse prognostic impact in the $A B C$ subtype but not the GCB subtype of DLBCL. (C) XPO1 ${ }^{\text {high }}$ expression showed a trend of unfavorable prognostic effect on PFS in MYC-rearranged $\left(M Y C-R^{+}\right)$ DLBCL. (D) XPO1 ${ }^{\text {high }}$ expression was associated with significantly poorer survival in DLBCL patients with wild type (Wt) TP53. (E) ABC-DLBCL and GCB-DLBCL cells showed similar sensitivity to the cytotoxicity of selinexor. (F) TP53 mutation (Mut-TP53) significantly reduced the anti-lymphoma efficacy of selinexor in HGBCL-DH cells. IC50 values were calculated by GraphPad Prism 8 based on the cell viability data after 72 -hour treatment.

\section{Abbreviations}

DLBCL: Diffuse large B-cell lymphoma; R/R: Relapsed or refractory; GCB: Germinal center B-cell-like; ABC: Activated B-cell-like; PFS: Progressive-free survival; OS: Overall survival; MYC-R: MYC rearrangement; $B C L 2-R$ : $B C L 2$ rearrangement; HGBCL-DH: High-grade B-cell lymphoma with MYC and BCL2 double-hit; Wt: Wild type; Mut: Mutant or mutated; BET: Bromodomain and extra-terminal domain.

\section{Acknowledgements}

Not applicable.

\section{Authors' contributions}

Conception and design were performed by MD, ZYXM, BX, and KHY. Research performance was performed by MD, MZ, ZYXM, LVP, BX, and KHY. Provision of study thought, materials, key reagents and technology were performed by MD, MZ, ZYXM, LVP, AT, CV, XF, GB, FZ, KD, AC, WT, YZ, EDH, WWLC, JH, MP, AJMF, MBM, BMP, JHvK, MAP, JNW, FH, LA, YL, MA, BX, and KHY. Collection and assembly of data under approved IRB and Material Transfer Agreement were done by MD, MZ, ZYXM, LVP, AT, CV, XF,GB, FZ, KD, AC, WT, YZ, EDH, WWLC, JH, MP, AJMF, MBM, BMP, JHVK, MAP, JNW, FH, LA, YL, MA, BX, and KHY. Data analysis and interpretation were performed by MD, MZ, ZYXM, LVP, BX, and KHY. Manuscript writing was performed by MD, ZYXM, BX, and KHY. Final approval of manuscript was performed by all authors who read and approved the final manuscript.

\section{Funding}

This work was supported in part by the Cancer Prevention and Research Institute of Texas, the Hagemeister Lymphoma Foundation and Gundersen Lymphoma Foundation.

Availability of data and materials

The datasets supporting the conclusions of this study are included in the figures and additional files.

\section{Ethics approval and consent to participate}

The study was approved by as being of minimal to no risk or as exempt by the institutional review board of each participating institution.

\section{Consent for publication}

Not applicable.

\section{Competing interests}

All authors declare no conflicts of interest.

\section{Author details}

${ }^{1}$ Department of Hematology, The First Affiliated Hospital of Xiamen University and Institute of Hematology, Xiamen University, School of Medicine, Xiamen, Fujian, China. ${ }^{2}$ Division of Hematopathology, Department of Pathology, Duke University Medical Center, Durham, NC 27710, USA. ${ }^{3}$ Department of Oncology, The First Affiliated Hospital of Zhengzhou University, Zhengzhou, China. ${ }^{4}$ Phamacyclics, an Abbvie Company, San Francisco, CA, USA. ${ }^{5}$ Institute of Pathology, University Hospital Basel, Basel, Switzerland. ${ }^{6}$ Department of Medicine, Section of Hematology, University of Verona, Verona, Italy. ${ }^{7}$ Columbia University Medical Center and New York Presbyterian Hospital, New York, NY, USA. ${ }^{8}$ Aalborg University Hospital, Aalborg, Denmark. ${ }^{9}$ Mayo Clinic, Rochester, MN, USA. ${ }^{10}$ Weill Medical College of Cornell University, New York, NY, USA. ${ }^{11}$ The Methodist Hospital, Houston, TX, USA. ${ }^{12}$ Cleveland Clinic, Cleveland, OH, USA. ${ }^{13}$ University of Hong Kong Li Ka Shing Faculty of Medicine, Hong Kong, China. ${ }^{14}$ Asan Medical Center, Ulsan University College of Medicine, Seoul, Korea. ${ }^{15}$ San Raffaele H. Scientific Institute, Milan, Italy. ${ }^{16}$ Odense University Hospital, Odense, Denmark. ${ }^{17}$ Gundersen Lutheran Health System, La Crosse, WI, USA. ${ }^{18}$ Radboud University Nijmegen Medical Centre, Nijmegen, The Netherlands. ${ }^{19}$ Hospital Universitario Marqués de Valdecilla, Santander, Spain. ${ }^{20}$ Feinberg School of Medicine, Northwestern University, Chicago, IL, USA. ${ }^{21}$ Department of Lymphoma/Myeloma, The University of Texas MD Anderson Cancer Center, Houston, TX, USA. ${ }^{22}$ Division of Hematology, Department of Internal Medicine, The Ohio State University, Columbus, OH, USA. ${ }^{23}$ Department of Medicine, Baylor College of Medicine, Houston, TX, USA. ${ }^{24}$ Department of Leukemia, The University of Texas MD Anderson Cancer Center, Houston, TX, USA. ${ }^{25}$ Key Laboratory of Xiamen 
for Diagnosis and Treatment of Hematological Malignancy, Xiamen, China.

${ }^{26}$ Duke Cancer Institute, Durham, NC, USA.

Received: 19 September 2020 Accepted: 22 October 2020

Published online: 04 November 2020

\section{References}

1. Azizian NG, Li Y. XPO1-dependent nuclear export as a target for cancer therapy. J Hematol Oncol. 2020;13(1):61.

2. Gravina GL, Senapedis W, McCauley D, Baloglu E, Shacham S, Festuccia C. Nucleo-cytoplasmic transport as a therapeutic target of cancer. J Hematol Oncol. 2014;7:85.

3. Kalakonda N, Maerevoet M, Cavallo F, Follows G, Goy A, Vermaat JSP, et al. Selinexor in patients with relapsed or refractory diffuse large B-cell lymphoma (SADAL): a single-arm, multinational, multicentre, open-label, phase 2 trial. Lancet Haematol. 2020;7(7):e511-22.

4. Visco C, Li Y, Xu-Monette ZY, Miranda RN, Green TM, Li Y, et al. Comprehensive gene expression profiling and immunohistochemical studies support application of immunophenotypic algorithm for molecular subtype classification in diffuse large B-cell lymphoma: a report from the International DLBCL Rituximab-CHOP Consortium Program Study. Leukemia. 2012;26(9):2103-13.

5. Luo B, Huang L, Gu Y, Li C, Lu H, Chen G, et al. Expression of exportin-1 in diffuse large B-cell lymphoma: immunohistochemistry and TCGA analyses. Int J Clin Exp Pathol. 2018;11(12):5547-60.

6. Hu S, Xu-Monette ZY, Tzankov A, Green T, Wu L, Balasubramanyam $A$, et al. MYC/BCL2 protein coexpression contributes to the inferior survival of activated B-cell subtype of diffuse large B-cell lymphoma and demonstrates high-risk gene expression signatures: a report from The
International DLBCL Rituximab-CHOP Consortium Program. Blood 2013; 121(20):4021-31; quiz 4250

7. Jaffe ES, Barr PM, Smith SM. Understanding the new WHO classification of lymphoid malignancies: why it's important and how it will affect practice. Am Soc Clin Oncol Educ Book. 2017;37:535-46.

8. Xu-Monette ZY, Wu L, Visco C, Tai YC, Tzankov A, Liu WM, et al. Mutational profile and prognostic significance of TP53 in diffuse large B-cell lymphoma patients treated with R-CHOP: report from an International DLBCL Rituximab-CHOP Consortium Program Study. Blood. 2012;120(19):3986-96.

9. Xu-Monette ZY, Moller MB, Tzankov A, Montes-Moreno S, Hu W, Manyam GC, et al. MDM2 phenotypic and genotypic profiling, respective to TP53 genetic status, in diffuse large B-cell lymphoma patients treated with rituximab-CHOP immunochemotherapy: a report from the International DLBCL Rituximab-CHOP Consortium Program. Blood. 2013;122(15):2630-40.

10. Liu Y, Azizian NG, Dou Y, Pham LV, Li Y. Simultaneous targeting of XPO1 and BCL2 as an effective treatment strategy for double-hit lymphoma. J Hematol Oncol. 2019;12(1):119.

11. Fischer MA, Friedlander SY, Arrate MP, Chang H, Gorska AE, Fuller LD, et al. Venetoclax response is enhanced by selective inhibitor of nuclear export compounds in hematologic malignancies. Blood Adv. 2020;4(3):586-98.

12. Li W, Gupta SK, Han W, Kundson RA, Nelson S, Knutson D, et al. Targeting MYC activity in double-hit lymphoma with MYC and BCL2 and/or BCL6 rearrangements with epigenetic bromodomain inhibitors. J Hematol Oncol. 2019;12(1):73.

\section{Publisher's Note}

Springer Nature remains neutral with regard to jurisdictional claims in published maps and institutional affiliations.
Ready to submit your research? Choose BMC and benefit from:

- fast, convenient online submission

- thorough peer review by experienced researchers in your field

- rapid publication on acceptance

- support for research data, including large and complex data types

- gold Open Access which fosters wider collaboration and increased citations

- maximum visibility for your research: over 100M website views per year

At BMC, research is always in progress.

Learn more biomedcentral.com/submissions 